\title{
HIGH-ENERGY PARTICLES AND MACHINES FOR THEIR ACCELERATION
}

\begin{abstract}
A GROUP of papers on high-energy particles and machines for their acceleration was arranged by Section A (Mathematics and Physics) of the British Association, and presented at Birmingham on August 31. In opening the session, Prof. R. E. Peierls said that our interest in fast particles arises from the fact that we wish to learn more about the nature of the forces occurring in the atomic nucleus. In the past fifty years, the fundamental laws governing atomic structure have been well established. The experimental data upon which they are based was obtained by a careful study of the interaction between electromagnetic waves of suitable wave-lengths, that is, light and X-rays and the internal electric fields of the atom. The refinements introduced by the quantum theory were necessary to take account of the small distances over which these electric fields are acting.
\end{abstract}

To establish the nature of the forces within the nucleus a similar study is necessary; but the important difference arises that these forces are known to be not electric in nature. They are much stronger than electric forces and act over shorter distances, the nucleus being of the order of $10^{5}$ times smaller than the atom. To react strongly with these forces, bombarding projectiles of very high energies are necessary. For example, with more energetic particles than are yet available, we may hope to produce in the laboratory a meson heavier than the $\pi$-meson. There is some evidence for this particle in cosmic ray work. At about three times the highest energy yet achieved, negative protons may also be expected. They should occur in a similar way to the creation of positive and negative electrons by the interaction of electric fields. In addition, we must never overlook the possibility of now events occurring about which we know nothing at present.

Mr. D. W. Fry described recent progress with the travelling-wave linear accelerator method for producing high-energy electrons. The method employs the $E_{0}$-type electromagnetic wave, which may be built up in a cylindrical metal tube loaded with metal corrugations. The velocity of the wave and electrons injected into it may be made to remain in step as they travel along the accelerating tube. Radial defocusing fields may be overcome by applying a steady axial magnetic field. With an accelerator built at Malvern using a peak power of $2 \mathrm{MW}$. in 2 - $\mu$ sec. pulses on a wave-length of $10 \mathrm{~cm}$., electrons were accelerated to $3 \cdot 8 \mathrm{MeV}$. in a distance of 2 metres. The maximum accelerated pulse current was 300 m.amp. It is now being used at Harwell to generate a pulsed source of neutrons by the action of gammarays on beryllium. Neutrons of energies up to $1 \mathrm{keV}$. are available to study the properties of materials. A 15-MeV. accelerator to provide an even larger flux of high-energy neutrons is now being made. The main characteristics of a $10-\mathrm{MeV}$. machine being built for the Medical Research Council in Messrs. Metropolitan-Vickers Laboratories were also outlined. Mr. Fry said that the production of 1,000. $\mathrm{MeV}$. electrons by a linear accelerator is now being considered. Theoretical studies by Mr. Harvie and his colleagues show that the power required to accelerate electrons to this energy might be reduced substantially by using wave-guides suitably loaded with low-loss high-permittivity ceramic disks instead of the copper diaphragms used so far. Experimental work upon these new types of wave-guide has been started.

Dr. L. U. Hibbard then described the University of Birmingham 1,000-MeV. proton synchrotron. It is a cyclic accelerator in which the particles travel around a toroidal-shaped vacuum chamber at an approximately constant radius. This chamber is placed between the poles of an A.c. magnet, acceleration taking place when the magnetic guiding field threading the annulus is increasing. A radiofrequency field is produced within the annulus by a C-shaped electrode, and the energy of the particles increases if they pass through this field at a suitable phase. Automatic synchronism between the particles and the radio-frequency field is maintained so long as the radio-frequency is suitably adjusted with increasing magnetic field. In the Birmingham machine the orbit radius is $450 \mathrm{~cm}$. The magnetic field in the annulus rises to a maximum value of 15,000 gauss in one second. The radio-frequency accelerating voltage will be 240 volts r.m.s. produced between the ends of a C-shaped electrode stretching $130^{\circ}$ around the annulus and an outer electrode at earth potential. Protons will be injected at $0.5 \mathrm{MeV}$. and the radio-frequency changed from $800 \mathrm{kc} . / \mathrm{s}$. to $30 \mathrm{Mc} / \mathrm{s}$. during the acceleration of the particles. The magnet construction, excitation and cooling were described. To prevent an excessive loss of particles in the early stages by gas scattering, phase oscillations and other causes, the radio-frequency and the magnetic field must be accurately related. Also the working gas pressure must be not greater than $10^{-8} \mathrm{~mm}$. of mercury. The steps being taken to meet these requirements were described.

Experiments using the 25-MeV. electron synchrotron at the Atomic Energy Research Establishment, Harwell, were discussed by Mr. F. K. Goward. Nuclear emulsions have been irradiated with the gamma-ray beam and the nuclear explosions producing three- and four-pronged stars examined. The three-pronged stars are found to be the disintegration products of carbon nuclei ; the four-pronged ones are of oxygen nuclei. With carbon photodisintegration it is found that cne alpha-particle is first emitted, leaving a beryllium-8 nucleus which then disintegrates into two alpha-particles. The beryllium-8 nueleus may be left in either the ground-state or an excited level. Goward and Wilkins have found that a large fraction of the observed events pass through the $3-\mathrm{MeV}$. level, but some pass through the groundstate. This latter condition had not been previously reported. The cross-section versus energy for the reaction has been measured; it shows a sharp fall above $18 \mathrm{MeV}$. This may be due to competitive processes, in particular the $(\gamma, n)$ reaction. In the oxygen disintegration, it is found that the predominant mode of disintegration is via the $9 \cdot 7-\mathrm{MeV}$. excited level of carbon-12 and not by initial splitting into two beryllium- 8 atoms. The carbon-12 in turn splits into beryllium-8 nuclei, which then disintegrate into 
two alpha-particles. Mr. Goward believes that the importance of these experiments lies in the information which they should provide for checking up the alpha-particle model of nuclear structure and the advances in nuclear techniques which are being made in these studies.

Experiments with the frequency-modulated cyclotron at Harwell were described by Dr. T. G. Pickavance. This machine, which first operated in December 1949, makes use of the principle of phasestable particle orbits, described independently by Veksler and McMillan in 1945. It is designed to accelerate protons to a maximum energy of $175 \mathrm{MeV}$. The magnet poles are $110 \mathrm{in}$. in diameter and its power consumption $350 \mathrm{~kW}$. for a central field of 16,000 gauss. The accelerated mean beam current is 1.5 microamp.

The first experiments carried out have been the measurement of total collision cross-section of highenergy neutrons in hydrogen. A well-collimated neutron beam, produced by the $171-\mathrm{MeV}$. proton beam falling on an internal beryllium target, was detected by counting recoil protons from a polythene disk in the beam with a triple-coincidence telescope of three proportional counters. Absorbers in the telescope limited the effective energy of the detectable neutrons to $\mathbf{1 5 6} \mathrm{MeV}$. The beam intensity was compared with and without attenuating rods of polythene and carbon in it. The measured total cross-section was $4.64 \pm 0.12 \times 10^{-28} \mathrm{~cm} .^{2}$.

The energy spectra of neutrons ejected from beryllium, carbon and aluminium targets have also been measured and were shown. A triple-coincidence telescope was again used, the third counter being replaced with an ionization chamber gated to count protons over only a limited energy range. The energy spectrum was measured as a function of absorber thickness between counters for both polythene and carbon radiators. The transparency of beryllium, carbon and aluminium was found to decrease in that order ; so also did the yield of high-energy neutrons.

Prof. P. M. S. Blackett concluded the session by describing evidence from cloud-chamber studies of cosmic ray showers for the existence of two new particles. In December 1947, Rochester and Butler published two cloud-chamber photographs containing forked tracks of a striking character. It was their opirrion that one of the forked tracks represented the spontaneous transformation in the gas of the cloud chamber of a new type of uncharged particle into two lighter charged particles, and the other the transformation of a new type of charged particle into two light particles, one of which was charged and the other uncharged. The apparatus has since been taken to the top of the Pic du Midi, where the probability of finding further similar events should be increased by eighteen to twenty times. Six V-tracks have been found in the past two months. Prof. Blackett said he had also heard from Dr. C. Anderson of Pasadena that he has found thirty-four events having similar characteristics. Of Anderson's events, thirty may be interpreted as a neutral particle disintegrating into a positive and a negative particle, and four as a charged particle going to a lighter charged particle and a neutral one.

From preliminary analyses of the Pic du Midi photographs, it seems probable that the lighter particles are $\pi$-mesons, so that the disintegrations might be of the form :

$$
V_{0} \rightarrow \tau_{+}+r_{-} \text {or } V_{ \pm} \rightarrow \pi_{ \pm}+\pi_{0} .
$$

The energy of the new particle is in the region of $10^{\circ}$ electron volts, and Butler's preliminary estimate of the mass of his $\pi$-mesons, $700-800$, makes it rather lower than the present generally accepted figure. One photograph obtained might be open to interpretation as a double $V$-decay, but further evidence is required.

$$
\text { D. W. FRY }
$$

\section{FINGER PRINTS AND DISEASE}

\section{By F. R. CHERRILL}

Chief Superintendent-in-Charge, Finger Print Branch, Criminal Investigation Department, New Scotland Yard, London, S.W.I

$\Delta \mathrm{S}$ a result of examination of the hands of decomA posed cadavers over a long period, it has been noticed that as a rule the muscles and skin of the left hand exhibit signs of greater decomposition than the right. This may be explained by the fact that the muscles, tendons, aponeuroses, etc., are less developed in the left hand than in the right, and would, therefore, less effectively withstand the ravages of the elements.

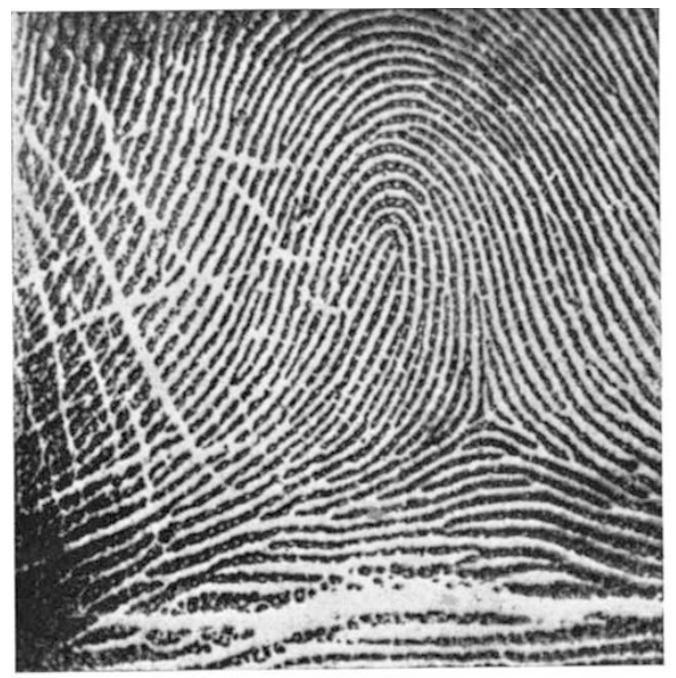

Fig. 1

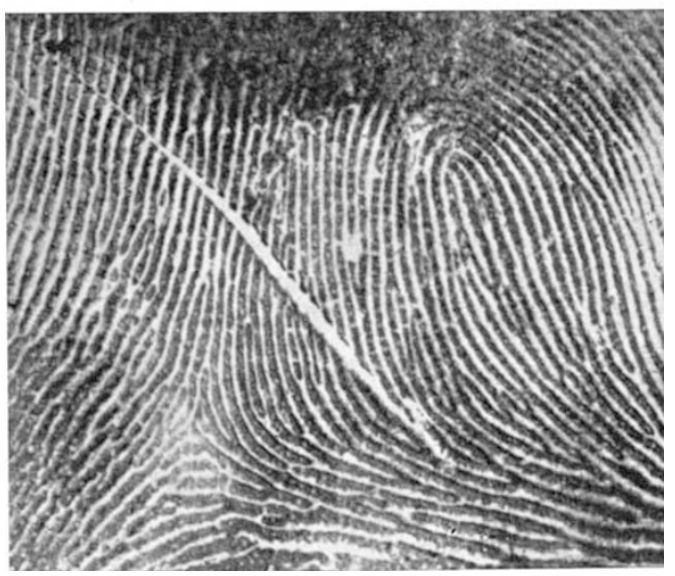

Fig. 2 